\title{
$\nabla$
}

\section{Hidradenitis suppurativa: epidemiological study of cases diagnosed at a dermatological reference center in the city of Bauru, in the Brazilian southeast State of São Paulo, between 2005 and 2015*}

\author{
Tatiana Cristina Pedro Cordeiro de Andrade ${ }^{1}$ \\ Agnes Mayumi Nakano Oliveira ${ }^{1}$ \\ Tatiane Meira Santiago ${ }^{1}$
}

\author{
Bruna Cortinóvis Vieira ${ }^{1}$ \\ Tábata Yamasaki Martins ${ }^{1}$ \\ Antônio Carlos Ceribelli Martelli ${ }^{1}$
}

DOI: http:/ / dx.doi.org/10.1590/abd1806-4841.20175588

\begin{abstract}
BACKGROUND: Hidradenitis is a chronic inflammatory disease of the hair follicles. A treatment is necessary due to chronicity and psychological changes that patient present.

Овјестіvе: To investigate epidemiological aspects and elaborate a risk group profile, promote early diagnosis and contribute to the knowledge about the disease.

METHODS: This cross-sectional descriptive study with retrospective analysis of medical records of 194 patients diagnosed with hidrosadenitis in a dermatological reference center in the city of Bauru (SP) between 2005 and 2015.

RESUlTS: Females accounted for $74 \%$ of cases. The age at diagnosis ranged from 10 to 67 years and the majority was within the $3 \mathrm{rd}$ and 4 th decade of life. It occurred Association with diabetes mellitus in $33 \%$, obesity in $55 \%$ and smoking in $61 \%$ was observed. Mean time between the onset of the disease and diagnosis was nine years. Hurley stage II was the most common at diagnosis. The therapeutic option mostly used in Hurley I and II was systemic antibiotics and in Hurley III was surgery.

STUDy LIMITATIONS: the main limitation of this study is its retrospective design, which does not allow the true clinical confirmation of the disease by investigators.

Conclusion: we outlined the following profile: women, caucasian, between 3rd and 4th decade of life, associated with obesity, smoking, late diagnosis and multiple potential therapeutic modalities. We highlight the importance of studies like this in order to identify risk groups and encourage early diagnosis.
\end{abstract}

Keywords: Epidemiology; Hidradenitis suppurativa; Hidradenitis

\section{INTRODUCTION}

Hidradenitis suppurativa (HS) is a chronic inflammatory debilitating disease of the hair folicles with a wide spectrum of severity. ${ }^{1-7}$ It is characterized by recurrent nodules, bridge scars and suppuration in intertriginous areas..$^{1,2,8,9}$ The disease severely affects patients' quality of life. ${ }^{1,9}$ When associated with acne conglobata, pilonidal cysts and dissecting cellulitis of the scalp, the syndrome is defined as a tetrad of follicular occlusion. ${ }^{1}$

As there are no diagnostic tests and the histology is not specific, the diagnosis of HS is based on 3 compulsory clinical criteria: typical clinical lesions (deep painful nodules, abscesses, bridge scars and double open comedones), typical location, (armpits, groin, perineal and perianal region, buttocks, infra-mammary), associated with recurrence and chronicity. ${ }^{1,5,8,9}$

We conducted a study that aimed at investigating epidemiological aspects not yet totally elucidated on hidradenitis suppurativa and to elaborate a profile corresponding to a risk group, to promote the early diagnosis and to contribute to the understanding of this dermatosis

\section{METHODS}

This is a cross-sectional and descriptive study with a retrospective analysis of medical records of all patients who had a clinical diagnosis of HS in tertiary referral public unit in dermatology in the city of Bauru (SP) between August 2005 and August 2015.

Sampling was of a non-probabilistic type for convenience, including all patients with a clinical diagnosis of HS during the study period, and for definitive diagnosis of HS patients should present the 3 mandatory criteria (typical lesion, typical site and recurrence). Patientes who did not fulfill these criteria were excluded. In total, 194 records were reviewed and 123 cases were selected to participate in this study.

For each case, the epidemiological and clinical characteristics were identified: age, sex, race, previous history of diabetes mellitus, smoking, obesity, association with acne and follicular occlusion tetrad, time between onset of lesion and diagnosis, Hurley stage and the treatment performed. Data as factors of worsening and family history were reported in few charts, so they could not be part of this analysis.

\footnotetext{
Received on 18.01.2016

Approved by the Advisory Board and accepted for publication on 14.03.2016

* Study conducted at Instituto Lauro de Souza Lima (ILSL) - Bauru (SP), Brazil.

Financial support: None.

Conflict of interest: None.

Department of Dermatology, Instituto Lauro de Souza Lima (ILSL) - Bauru (SP), Brazil.

(C2017 by Anais Brasileiros de Dermatologia
} 
Because this is a study based on data collection of medical records, possible biases of measurement and information should be considered.

Data obtained were processed in Microsoft ${ }^{\circledR}$ Excel, with frequency and percentage analysis, and this program was used for the elaboration of the graphs. The principles of the Helsinki Declaration were respected during the study.

\section{RESULTS}

In this study, $74 \%$ of the cases were women and $26 \%$ were men. The women:men ratio was $2.8: 1 ; 76 \%$ were Caucasian, $18 \%$ were non-Caucasian and in $6 \%$ this was not described in the chart (Figure 1). Age at diagnosis ranged from 10 to 67 years, with most of them in the third (25.2\%) and fourth (26.8\%) decade of life (Figure 2). Presence of comorbidities was analyzed and the association with diabetes mellitus occurred in $33 \%$ of the patients; obesity in $55 \%$ and smoking in $61 \%$ of patients but, in $33 \%$ of cases the data were not available. Presence of follicular occlusion tetrad was observed in $17 \%$ of patients. Also, acne was present in $32 \%$ of subjects (Figure 3). Mean time between disease onset and diagnosis was 9 years, but most of the patients were diagnosed within 4 years of onset of symptoms, with the shortest time for diagnosis being 9 months and the longest 32 years. Hurley stage at diagnosis varied greatly: $21 \%$

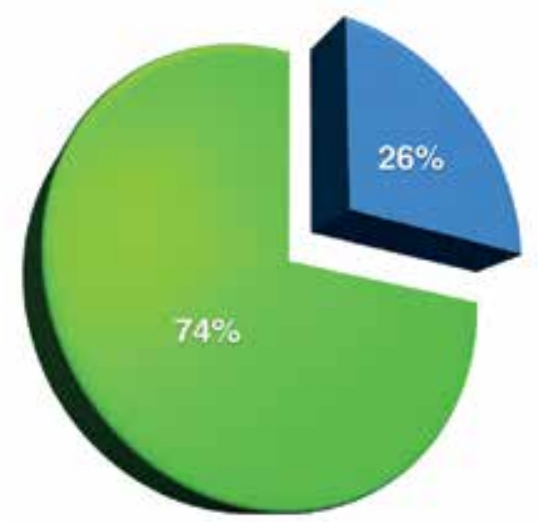

\section{Male Female}

FIGURE 1 : Distribution of cases of hidradenitis suppurativa according to gender

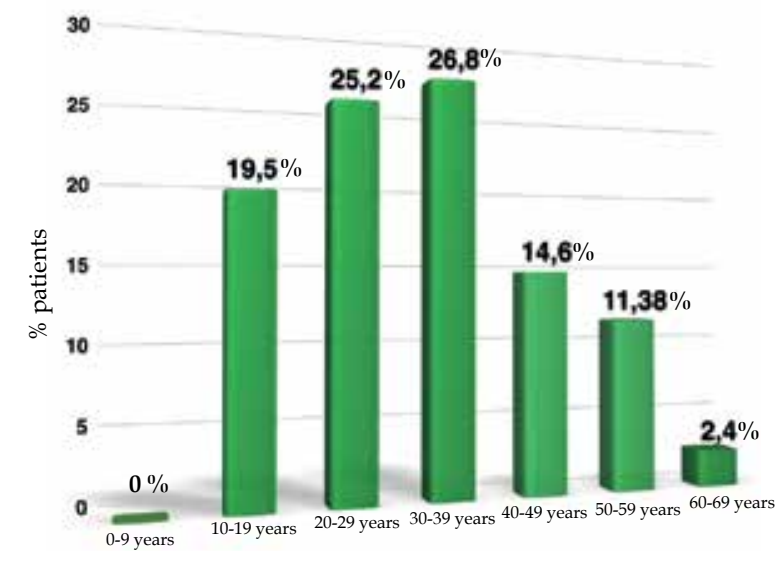

Figure 2: Demonstration of the age at the diagnosis of hidradenitis suppurativa, distributed in age groups presented Hurley I; 48\% presented Hurley II; and 31\% presented Hurley III (Figure 4). Regarding the various treatment modalities performed, they were divided into topical antibiotic alone (benzoyl peroxide and clindamycin were the most used); systemic antibiotic alone (tetracycline, doxycycline, dapsone and clindamycin were the most used); topical antibiotic associated with systemic antibiotic, isotretinoin and surgery. The most used option in patients with Hurley I and II was systemic antibiotic: $52 \%$ and $42 \%$, respectively; and in patients with Hurley III it was surgery with extensive excision, performed in $84 \%$ of cases (Figure 5).

\section{DISCUSSION}

Hidradenitis suppurativa, also called inverse acne, was described by Verneuil in $1854,{ }^{10,11}$ it has a prevalence reported in the literature of $0.3 \%$ to $4 \%$ of the population, but it is underestimated, since many patients report avoiding the physician for feeling ashamed due to the clinical condition. ${ }^{1,2,5,9-14}$ This data justifies its late diagnosis and in more advanced stages, as found in our study, with a mean time of 9 years for diagnosis and most patients presenting Hurley stage II or III.

Hurley described in 1989 a clinical severity score for HS and defined 3 stages of evolution: Hurley I, single abscesses or multiple abscesses without scarring or fistulae; Hurley II, recurrent abscesses with formation of scarring and bridges; and Hurley III, multiple abscesses, scarring and communicating fistulas involving the entire anatomical area (Figure 6). ${ }^{8}$

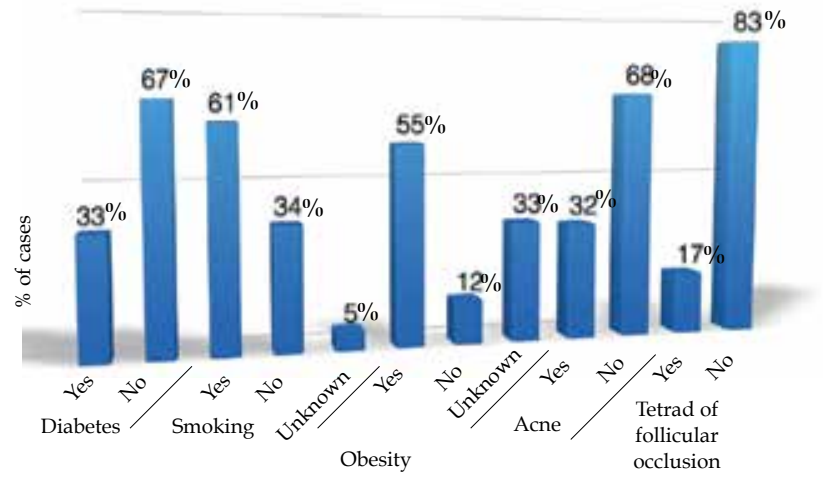

Figure 3 : Comorbidities and risk factors associated with patients diagnosed with hidradenitis suppurativa in this study

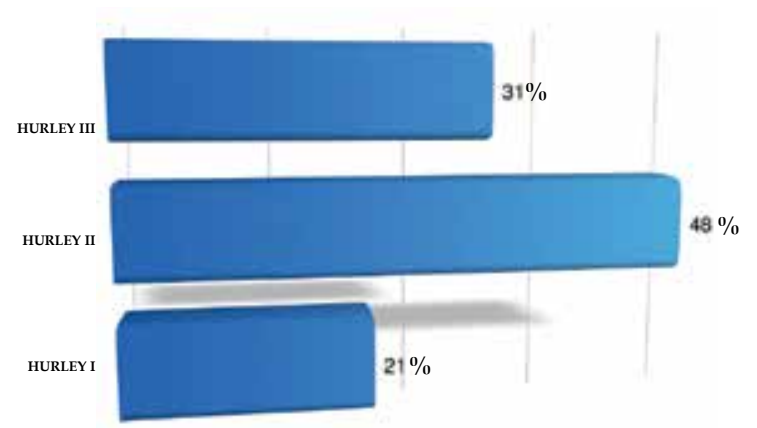

Figure 4 : Distribution of patients with hidradenitis suppurativa according to the Hurley stage 


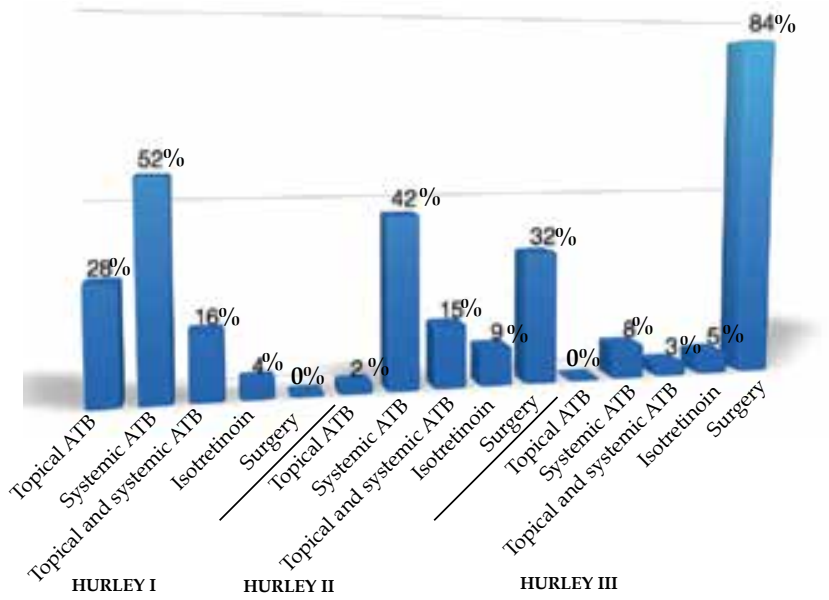

Figure 5 : Therapeutic modalities used according to Hurley stage. (* ATB: antibiotic)
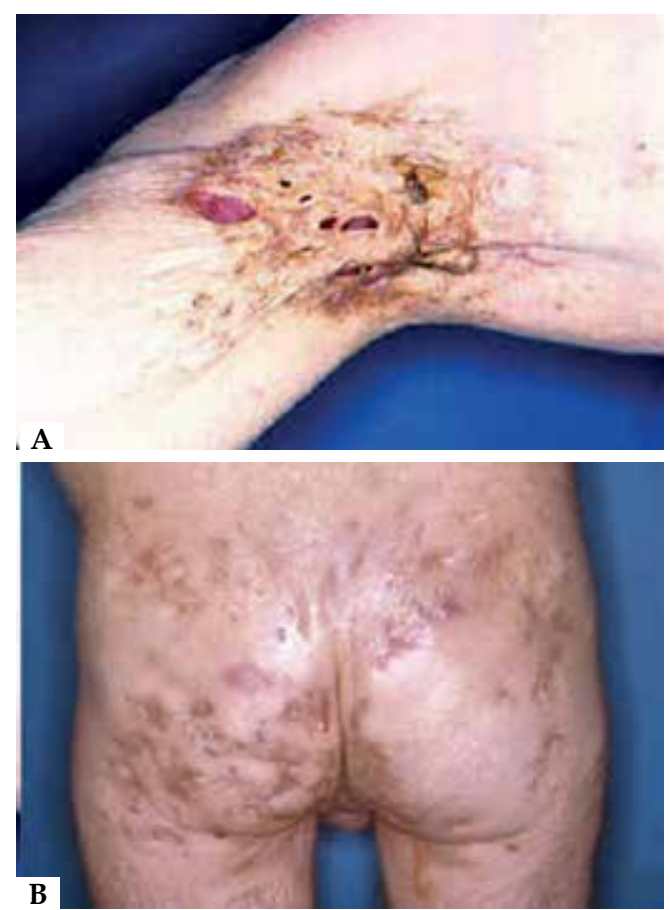

Figure 6 : Hidradenitis suppurativa in Hurley stage III: multiple abscesses, scarring and communicating fistulas involving all anatomical area in axilla and buttock

Studies report that women are more affected than men with a woman:man ratio described of 3:1,2,5,9-14 similar to the data found in our study. Vlassova et al. describe a predilection for African-descendant involvement, however, in this study we found a majority of caucasian patients. ${ }^{1}$ This may be justified because the two studies were performed in a single institution and the attendance population of those institutions may be divergent.

Its pathophysiology is partially defined and there are several theories that try to explain this disease; some authors believe that the primary defect is the occlusion of the hair follicle and subsequent infection of the apocrine glands, as the apocrine glands only begin their secretion after puberty the disease would begin after this period, and it is reported a higher frequency in the third decade of life as we could observe in our study. ${ }^{1,2,4}$ Others defend the theory that follicular occlusion would occur initially with posterior rupture of the hair follicle and formation of important immune reaction of the foreign body type, which would lead to all clinical alterations presented by these patients, not being associated with infection nor apocrine sweat glands. ${ }^{15}$ Recent studies on the immune pattern in HS varies among authors who believe in innate immunity deficiency to an exaggerated immune response. In the area of genetics it is believed that the Notch protein plays a significant role in the normal development of the hair follicle, and when altered it is associated with the formation of cysts. In addition, Notch is also an important modulator of T cell-mediated immune responses by suppressing pro-inflammatory responses. Thus changes in the inherited or acquired Notch may play a key role in the development of HS. ${ }^{15}$ Considerable progress has been made in HS research, but many aspects of its pathogenesis and inflammatory mediators involved are still being studied. ${ }^{15}$

HS is usually associated with some risk factors such as obesity, as well as diabetes mellitus (DM) and smoking. 2,4,5,7,-14,16 Kromann et al. estimate that the prevalence of HS in the obese population is $18.1 \%$, and that weight loss would lead to an improvement in severity. ${ }^{3}$ In Scheinfeld review, acne vulgaris has no relation to HS compared with normal controls. ${ }^{12}$ Kohorst et al. conducted a major review of factors related to HS and reported that obesity rates in HS patients ranged from $12 \%$ to $88 \%$, DM ranged from $5 \%$ to $20 \%$, smoking from $40 \% 92 \%$, follicular occlusion tetrad in $6 \%$, and acne vulgaris from $13 \%$ to $36 \% .^{7}$ This was similar to the data found in our study, except for DM, which occurred in 33\% of the cases, when compared with the literature reviewed and the tetrad of follicular occlusion that occurred in $17 \%$ of the cases in this study - a higher value that may be justified by our service be a tertiary care and receive patients with more debilitating pathologies.

Adequate treatment of HS is necessary because of the various psychological changes that patients undergo and its chronic character. $^{11,12,13}$ And this is the great difficulty of this pathology, since there are not many controlled and randomized studies for its definition; nevertheless, there is concordance among the authors of which surgical approach is the best option for patients in advanced stage and that systemic antibiotics present the best evidence among the options of systemic therapy in the treatment of HS. ${ }^{5,6,10}$ In our study we were able to observe the most commonly used therapeutic modality in each Hurley stage and we observed that the surgery approach was the first option in most Hurley III patients and systemic antibiotics were first choice in Hurley I and II, being in agreement with the exposed above.

The description of therapeutic modality data related to Hurley stage was not found in the reviewed literature and they were added by allowing a broad view of the therapy chosen by specialists for each stage; however, we reinforced the need for population-based and comparative studies to define the best therapy to be employed at each stage of HS.

The main limitation of this study is its retrospective design. Although the patients' charts were reviewed in an attempt to confirm the diagnosis of HS, this design does not allow true clinical confirmation by the investigators. 


\section{CONCLUSION}

In this study, the following profile was observed for HS: women, caucasian, between 3rd and 4th decades of life, associated with obesity and smoking, late diagnosis in advanced stage in most cases, and multiple possible therapeutic modalities. The importance of Studies like this, which identifies risk groups help to the knowledge of this disease and favor the early diagnosis. We emphasize the need for population-based studies to define the best therapy for each stage of the diasease.

\section{REFERENCES}

1. Vlassova N, Kuhn D, Okoye GA. Hidradenitis Suppurativa Disproportionately Affects African Americans: A Single-center Retrospective Analysis. Acta Derm Venereol. 2015;95:990-1.

2. Riis PT, Søeby K, Saunte DM, Jemec GB. Patients with hidradenitis suppurativa carry a higher systemic inflammatory load than other dermatological patients. Arch Dermatol Res. 2015;307:885-9.

3. Kromann CB, Ibler KS, Kristiansen VB, Jemec GB. The influence of body weight on the prevalence and severity of hidradenitis suppurativa. Acta Derm Venereol. 2014;94:553-7

4. Schmitt JV, Bombonatto G, Martin M, Miot HA. Risk factors for hidradenitis suppurativa: a pilot study. An Bras Dermatol. 2012;87:936-8.

5. Mehdizadeh A, Hazen PG, Bechara FG, Zwingerman N, Moazenzadeh M, Bashash $M$, et al. Recurrence of hidradenitis suppurativa after surgical management: $A$ systematic review and meta-analysis. J Am Acad Dermatol. 2015;73:S70-7.

6. Alhusayen $\mathrm{R}$, Shear $\mathrm{NH}$. Scientific evidence for the use of current traditional systemic therapies in patients with hidradenitis suppurativa. J Am Acad Dermatol. 2015;73:S42-6.

7. Kohorst JJ, Kimball AB, Davis MD. Systemic associations of hidradenitis suppurativa. J Am Acad Dermatol. 2015;73:S27-35.

8. van der Zee $\mathrm{HH}$, Jemec GB. New insights into the diagnosis of hidradenitis suppurativa: Clinical presentations and phenotypes. J Am Acad Dermatol. 2015;73:S23-6.

9. Zouboulis CC, Del Marmol V, Mrowietz U, Prens EP, Tzellos T, Jemec GB. Hidradenitis Suppurativa/Acne Inversa: Criteria for Diagnosis, Severity Assessment, Classification and Disease Evaluation. Dermatology. 2015;231:184-90.

10. Jemec GB, Kimball AB. Hidradenitis suppurativa: Epidemiology and scope of the problem. J Am Acad Dermatol. 2015;73:S4-7.

11. Muzy G, Crocco El, Alves RO. Hidradenite supurativa: atualização e revisão de suas modalidades terapêuticas. Surg Cosmet Dermatol 2014;6:206-12.

12. Scheinfeld N. Diseases associated with hidranitis suppurativa: part 2 of a series on hidradenitis Dermatol Online J. 2013;19:18558.

13. Miller IM, McAndrew RJ, Hamzavi I. Prevalence, Risk Factors, and Comorbidities of Hidradenitis Suppurativa. Dermatol Clin. 2016;34:7-16.

14. Dessinioti C, Katsambas A, Antoniou C. Hidradenitis suppurrativa (acne inversa) as a systemic disease. Clin Dermatol. 2014;32:397-408.

15. Prens E, Deckers I. Pathophysiology of hidradenitis suppurativa: An update. J Am Acad Dermatol. 2015;73:S8-11.

16. Shlyankevich J, Chen AJ, Kim GE, Kimball AB. Hidradenitis suppurativa is a systemic disease with substantial comorbidity burden: a chart-verified casecontrolanalysis. J Am Acad Dermatol. 2014;71:1144-50.

\author{
MAILING ADDRESS: \\ Tatiana Cristina Pedro Cordeiro de Andrade \\ Rua Dr. Plinio Barreto 173, apto 21A, Bela Vista \\ 01313-020 - São Paulo - SP \\ Brazil \\ Email: tatianap.andrade@gmail.com
}

How to cite this article: Andrade TCPC, Vieira BC, Oliveira AMN, Martins TY, Santiago TM, Martelli ACC. Hidradenitis suppurativa: epidemiological study of cases diagnosed at a dermatological reference center in the city of Bauru, in the Brazilian southeast State of São Paulo, between 2005 and 2015. An Bras Dermatol. 2017;92(2):196-9. 